\title{
Traumatic intracranial internal carotid artery dissection and pseudoaneurysm presenting as oculomotor nerve palsy
}

\author{
Jae Rim Kim, Dae-Won Seo
}

Department of Neurology, Samsung Medical Center, Sungkyunkwan University School of Medicine, Seoul, Korea

Received: January 18, 2021

Revised: April 25, 2021

Accepted: May 3, 2021

Corresponding author:

Dae-Won Seo

Department of Neurology, Samsung Medical Center, Sungkyunkwan University School of Medicine, 81 Irwonro, Gangnam-gu, Seoul 06351, Korea

Tel: +82-2-3410-3595

E-mail:daewon@skku.edu

\section{ABSTRACT}

The diagnosis of internal carotid artery dissection after trauma is often delayed or missed due to other injuries, leading to potentially permanent neurologic deficits, or even death. Early diagnosis and treatment can prevent devastating consequences. In this report, we present a case of delayed onset but rapidly progressive traumatic intracranial internal carotid artery dissection to emphasize the timing of magnetic resonance imaging with angiography.

Keywords: Aneurysm, false; Carotid arteries; Dissection
This is an Open Access article distributed under the terms of the Creative Commons Attribution Non-Commercial License (https:// creativecommons.org/licenses/ by-nc/4.0/).

\section{INTRODUCTION}

Traumatic internal carotid artery dissection (ICAD) is rare [1]. It usually occurs at the level of the 1st and 2nd cervical vertebraes, which are the most movable segments of the carotid arteries [2]. However, the intracranial segment can also be involved. Traumatic ICAD is more commonly reported in Asians than in other ethnic groups [3]. ICAD can present with stenosis, complete occlusion, or false aneurysm formation. False-aneurysms are reported in $13 \%$ to $49 \%$ of all ICADs [4].

The diagnosis of traumatic dissection is often delayed or missed due to co-existing and life threatening injuries and/or symptoms, leading to devastating consequences. Although the golden standard for identifying dissection is digital subtraction angiography (DSA), computed tomography angiography (CTA) is the preferred initial screening test [5]. However, DiCocco et al. [6] reviewed blunted patients who underwent CTA or DSA for screening and discovered $6 \%$ of the patients with normal CTA had cerebrovascular injuries found on DSA. Recently, endovascular and surgical managements have been found to be effective treatments for cerebral artery dissection. Therefore, early recognition and management are important issues.

In this report, we discuss a case of delayed but rapidly progressive intracranial ICAD after a motor vehicular accident. 


\section{CASE REPORT}

A 56-year-old woman presented to the emergency department with right mandibular swelling after a car accident. She was seated in the passenger seat when she bumped her right chin and anterior neck against the dashboard of the car. Her past medical history revealed a history of liver transplantation due to alcoholic liver cirrhosis and diabetes. Her liver function was normal, but routine blood analysis revealed a bleeding tendency. Her platelet count was $60,000 / \mu \mathrm{L}$. Prothrombin time (international normalized ratio [INR]) and activated partial thromboplastic time were prolonged (42.5 seconds [INR of 4.22] and 53.0 seconds). She was mildly drowsy but was able to describe her complaints. There was no neurological complaint and the neurological examination was unremarkable, except for the drowsiness. Initial blood pressure was $99 / 54 \mathrm{~mm} \mathrm{Hg}$, but after 1 hour abruptly dropped to $68 / 41 \mathrm{~mm} \mathrm{Hg}$.

Brain computed tomography (CT) on admission revealed a subdural hemorrhage in the left frontal area (Fig. 1A). Ultrasonography and diagnostic ascites tapping revealed hemoperitoneum. She was admitted by the general surgery department, underwent emergency surgery and was cared for in the intensive care unit. After surgery, she had fluctuating mental status but her clinical course improved.

However, on the 5th hospital day (HD), she suddenly developed right ptosis and proptosis with limitation of eye adduction. The right pupil was dilated and non-reactive to light. Brain CT and angiography were again performed but there was no interval change in hemorrhage. No aneurysm or stenosis were found (Fig. 1B, C). On the 6th HD, she was stupor- ous with right ophthalmoplegia and left hemiparesis. Brain magnetic resonance imaging (MRI) performed 20 hours after the previous $\mathrm{CT}$ revealed a pseudo-lumen in the right intracranial internal carotid artery (ICA) and an acute cerebral infarction in the right anterior choroidal artery territory (Fig. 2A, B). Pseudoaneurysm was multi-lobar with a diameter of $10.1 \mathrm{~mm}$ (Fig. 2C). It was in the interpeduncular cistern, compressing the right optic nerve posteriorly and the oculomotor nerve superiorly and medially (Fig. 2D). Pseudo-lumen and intimal flaps were observed up to the middle and anterior cerebral arteries (Fig. 2E, F). On the 7th HD, the patient was comatose. Final brain CT revealed massive subarachnoid and intracranial hemorrhages (Fig. 3). On the 10th HD, the patient was brain dead.

Based on the clinical and radiographic findings, the patient was diagnosed with dissection of the communicating portion of the right ICA with pseudoaneurysm formation and compressive neuropathies of the oculomotor and optic nerves.

\section{DISCUSSION}

Traumatic internal carotid artery dissection (TICAD) is relatively uncommon, but it can be life threatening. TICAD can be caused by blunt or penetrating injuries. The incidence of traumatic carotid dissection was estimated to be $0.08 \%$ [5]. The mechanism of TICAD is from a direct impact on the anterolateral neck or elongation of the artery due to hyperextension and rotation of the neck.

As seen in our case, TICAD can be followed by pseudoaneurysm. The changes in size of the aneurysm are variable. Some aneurysms (3\%) increase in size, but others may remain un-
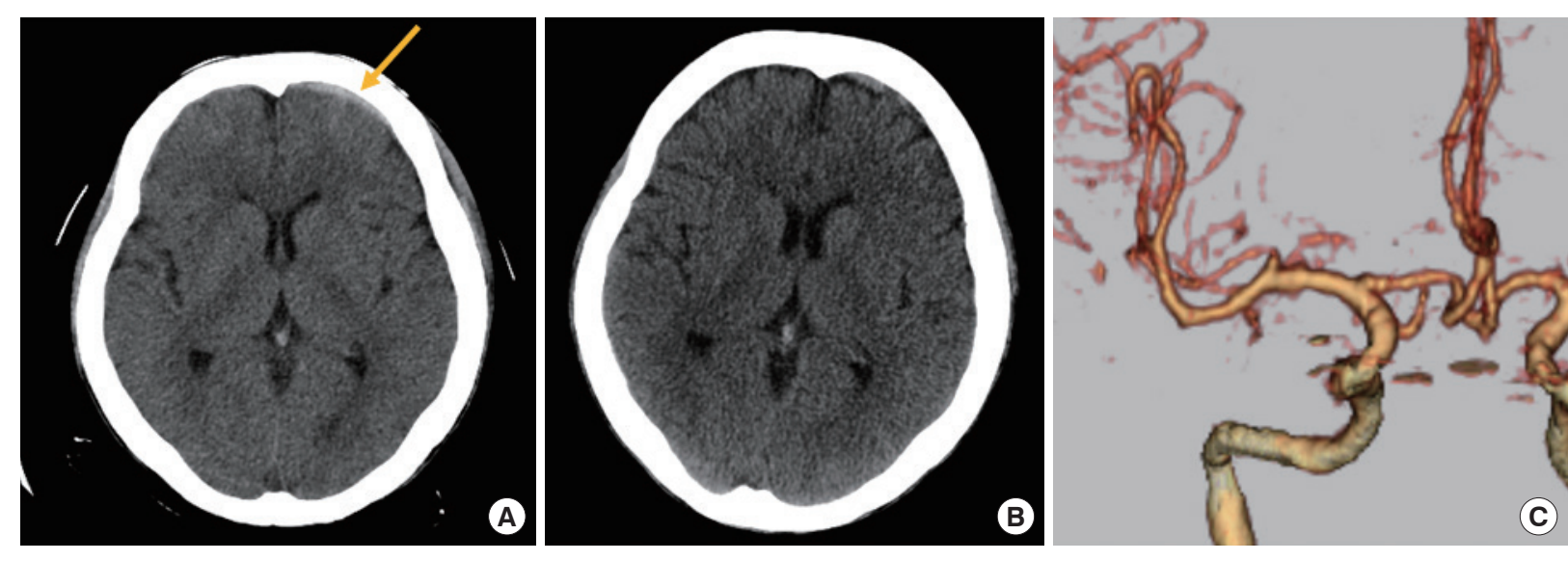

Fig. 1. Sequential brain computed tomography (CT) and angiography. (A) Initial CT shows an acute subdural hemorrhage along the left frontal convexity (arrow). (B) Follow-up CT does not show interval change 5 days after head trauma. (C) CT angiography shows no significant abnormalities in the intracranial vessels after oculomotor nerve palsy. 

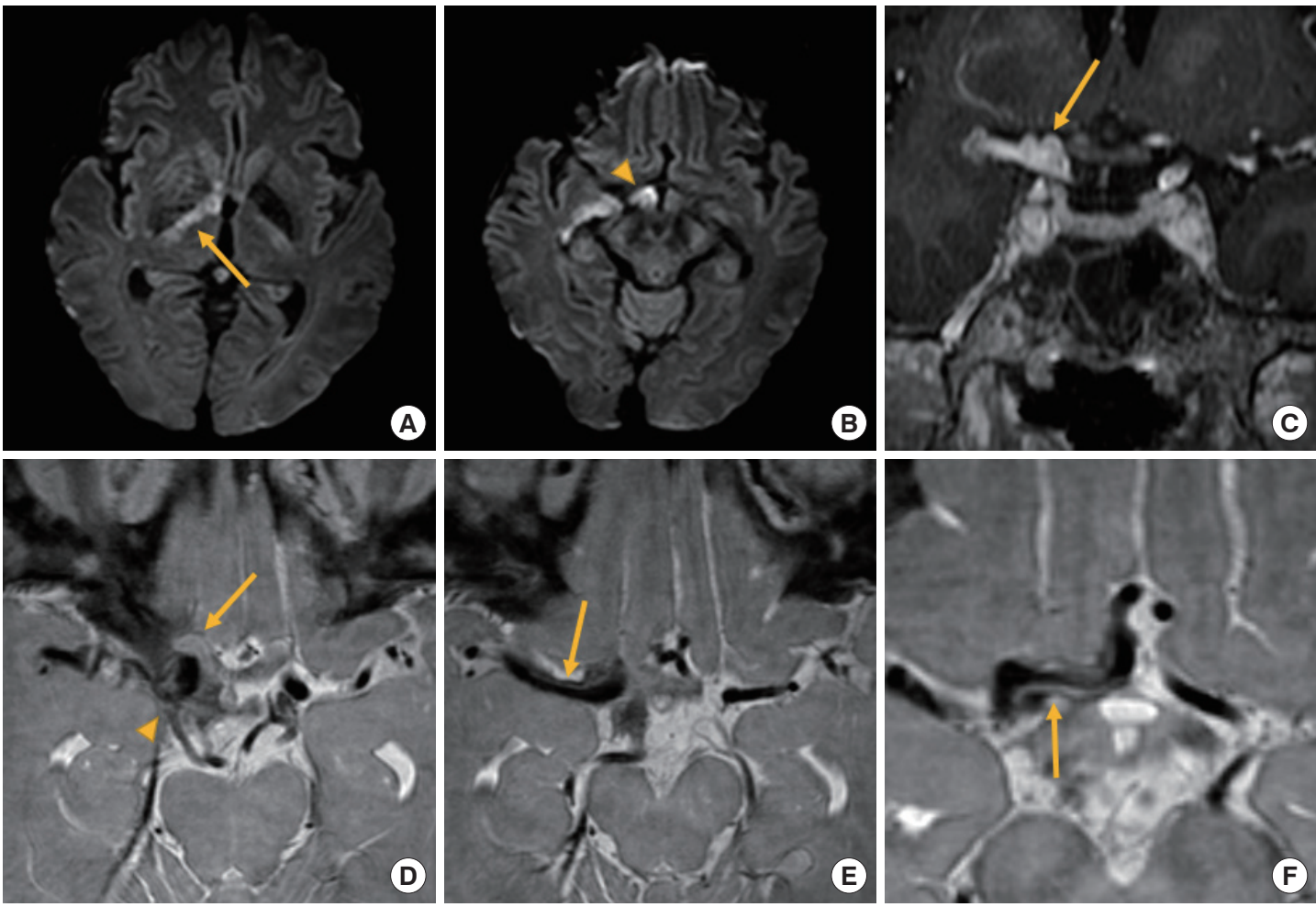

Fig. 2. Brain magnetic resonance imaging. (A, B) Diffusion-weighted imaging shows acute infarction in right anterior choroidal artery territory including the right posterior limb of internal capsule (arrow) and the optic nerve (arrowhead). (C) On T1-weighted image with contrast, pseudoaneurysm is multi-lobar (arrow) and located in the interpeduncular cistern. (D) On proton density-weighted imaging, the right optic (arrow) and oculomotor nerve (arrowhead) are compressed by pseudoaneurysm. (E, F) Pseudo-lumen (arrows) progressed to the right anterior and middle cerebral arteries.
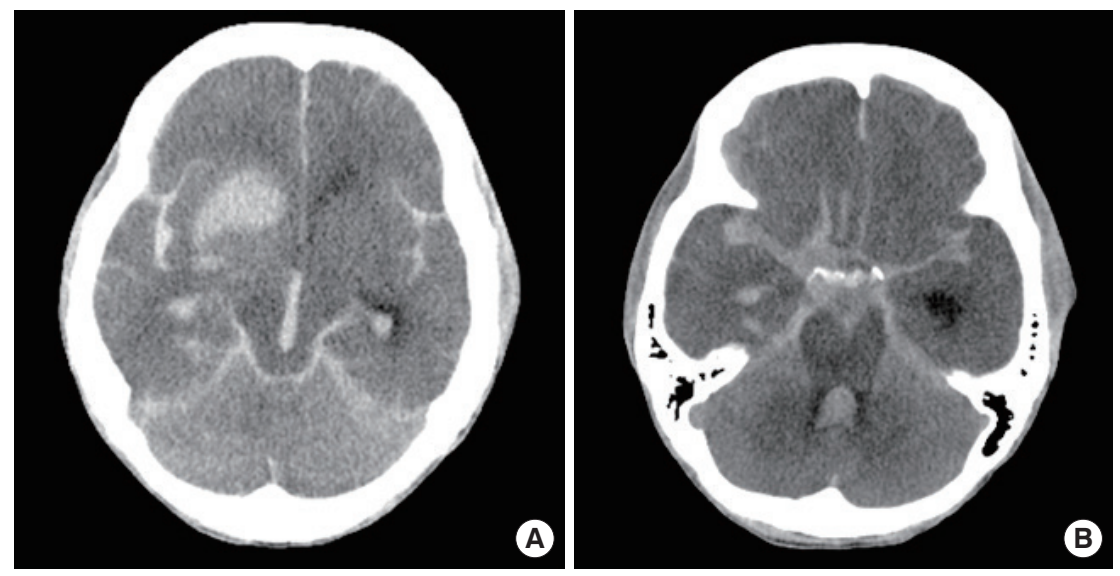

Fig. 3. Brain computed tomography. (A, B) Seven days later, computed tomography revealed massive subarachnoid and intracerebral hemorrhages.

changed or may even regress [4]. Pseudoaneurysms can cause complications such as distal thromboembolisms and compressions on surrounding structures [7]. Symptoms vary depending on the location. The lower cranial nerves are involved when the petrous segment of the ICA is affected [7]. However, when the affected segment of the ICA is above the cavernous segment, abnormal eye movements may be detected, as seen in our case.

Approximately $10 \%$ to $50 \%$ of TICADs present with abrupt symptoms within the first 24 hours after trauma [1,5]. However, symptoms may be delayed. Thus, if abnormal neurologic signs are seen any time after trauma, TICAD should be 
suspected. The most frequent manifestations include neck or head pain, stroke, Horner syndrome, and paralysis of the cranial nerves [5]. In contrast to spontaneous ICAD, patients with trauma often have several injuries, and the mental status is frequently altered. Therefore, a regular neurological monitoring is needed for early detection of focal deficits for a timely diagnosis and prompt management.

The gold standard for confirming dissection is DSA; however, it is an invasive and time-consuming test. Therefore, CTA is recommended as the initial screening modality [5]. In traumatic patients with symptoms or signs of injuries around the neck, screening CTA is important for early diagnosis and treatment of arterial dissection [1]. However, CTA sometimes fails to detect cerebrovascular injuries [6]. MRI with angiography takes longer than CTA but is also a useful diagnostic tool for TICAD. In particular, T1 weighted images and T1 weighted images with contrast media are useful to detect pseudolumen and intramural hematoma [8]. However, conventional slice MRI is too thick to delineate the intimal flap or intramural hematoma. Therefore, thin-slice images are recommended to detect intracranial artery dissection. There were even cases where pseudoaneurysm identified by magnetic resonance angiography were missed by CTA [9].

In our case, initial right pupil dilation without light reflex, ptosis, and limited eye adduction suggested oculomotor nerve palsy caused by external compression, which the most commonly due to an aneurysm. Proptosis may have occurred because of venous engorgement of the orbit. CTA performed after oculomotor nerve palsy was normal. However, the patient presented with rapid worsening of abnormal eye movements even though the CTA was normal. Brain MRI performed after CTA showed significant intracranial ICAD and pseudoaneurysm formation. If MRI had been performed earlier, she might have been able to receive prompt treatment.

In conclusion, TICAD may occur later after trauma but it can worsen quickly. CTA may show unremarkable results despite the presence of focal neurologic deficits, such as in this case. Therefore, it is recommended to undergo MRI with angiography for early detection of intracranial ICAD if there is worsening of neurologic symptoms despite normal CTA.

\section{CONFLICTS OF INTEREST}

This work was supported by Dong-A ST (No. PHO0162051).

\section{ORCID}

Jae Rim Kim https://orcid.org/0000-0002-3404-199X

Dae-Won Seo https://orcid.org/0000-0002-9266-9355

\section{AUTHOR CONTRIBUTIONS}

Conception or design: JRK, DWS.

Acquisition, analysis, or interpretation of data: JRK.

Drafting the work or revising: JRK.

Final approval of the manuscript: JRK, DWS.

\section{REFERENCES}

1. Agarwal A, Yadav D, Gupta A, Vishnu VY, Rajan R, Singh MB, et al. Delayed bilateral internal carotid artery dissection following motor vehicle accident: time to make its screening a part of trauma protocol? QJM 2020;113:672-3.

2. Lleva P, Ahluwalia BS, Marks S, Sahni R, Tenner M, Risucci DA, et al. Traumatic and spontaneous carotid and vertebral artery dissection in a level 1 trauma center. J Clin Neurosci 2012;19:1112-4.

3. Debette S, Compter A, Labeyrie MA, Uyttenboogaart M, Metso TM, Majersik JJ, et al. Epidemiology, pathophysiology, diagnosis, and management of intracranial artery dissection. Lancet Neurol 2015;14:640-54.

4. Paraskevas KI, Batchelder AJ, Naylor AR. Fate of distal false aneurysms complicating internal carotid artery dissection: a systematic review. Eur J Vasc Endovasc Surg 2016;52:281-6.

5. Galyfos G, Filis K, Sigala F, Sianou A. Traumatic carotid artery dissection: a different entity without specific guidelines. Vasc Specialist Int 2016;32:1-5.

6. DiCocco JM, Emmett KP, Fabian TC, Zarzaur BL, Williams JS, Croce MA. Blunt cerebrovascular injury screening with 32-channel multidetector computed tomography: more slices still don't cut it. Ann Surg 2011;253:444-50.

7. Cruciata G, Parikh R, Pradhan M, Shah J, Greif E, Stein EG. Internal carotid artery dissection and pseudoaneurysm formation with resultant ipsilateral hypoglossal nerve palsy. Radiol Case Rep 2017;12:371-5.

8. Kanoto M, Hosoya T. Diagnosis of intracranial artery dissection. Neurol Med Chir (Tokyo) 2016;56:524-33.

9. Hanning U, Sporns PB, Schmiedel M, Ringelstein EB, Heindel W, Wiendl $\mathrm{H}$, et al. CT versus MR techniques in the detection of cervical artery dissection. J Neuroimaging 2017;27:607-12. 